Highly Energetic Physical Processes and

Mechanisms for Emission from Astrophysical Plasmas

IAU Symposium, Vol. 195, 2000

P. C. H. Martens, S. Tsuruta, and M. A. Weber, eds.

\title{
Collimation of Magnetically Driven Outflows from Accretion Disks
}

\author{
T. Kudoh
}

Solar Physics Division, National Astronomical Observatory, Mitaka, Tokyo 181-8588, Japan

R. Matsumoto

Department of Physics, Faculty of Science, Chiba University, Inageku, Chiba 263-8522, Japan

K. Shibata

Kwasan and Hida Observatories, Kyoto University, Yamashina, Kyoto 607-8471, Japan

\begin{abstract}
We performed 2.5-dimensional, nonsteady MHD numerical simulations to investigate the acceleration and collimation of magnetically driven outflows from accretion disks, including the accretion process itself, consistently. As an initial condition, we used a paraboloidal magnetic field line that is produced by electric current on the equatorial plane. We found that the outflow ejected from the accretion disk is collimated by the pinch effect of the toroidal component of the magnetic field that is produced by the rotation of the disk.
\end{abstract}

\section{Introduction}

The magnetically driven jet is one of the exciting topics of high-energy astrophysics (Kudoh \& Shibata 1995, 1997a, 1997b). Previous MHD numerical simulations of magnetically driven jets from accretion disks concentrated on the acceleration mechanism of MHD jets under the assumptions of uniform magnetic fields and an isothermal corona as initial conditions (Uchida \& Shibata 1985; Shibata \& Uchida 1986; Matsumoto et al. 1994; Kudoh, Matsumoto, \& Shibata 1998). In this paper, the initial conditions of uniform magnetic fields and isothermal corona are relaxed in order to investigate the collimation mechanism of MHD jets in a large computational region. We used a nonuniform potential magnetic field as an initial condition, i.e., a paraboloidal magnetic field line that is produced by electric current on the equatorial plane. Ideal MHD is assumed under point-mass gravitation without disk self-gravity.

\section{Results}

Figure 1a shows the color density map and magnetic field lines after about five rotations of a Keplerian disk at $(r, z)=(1,0)$. The red line shows a stream 

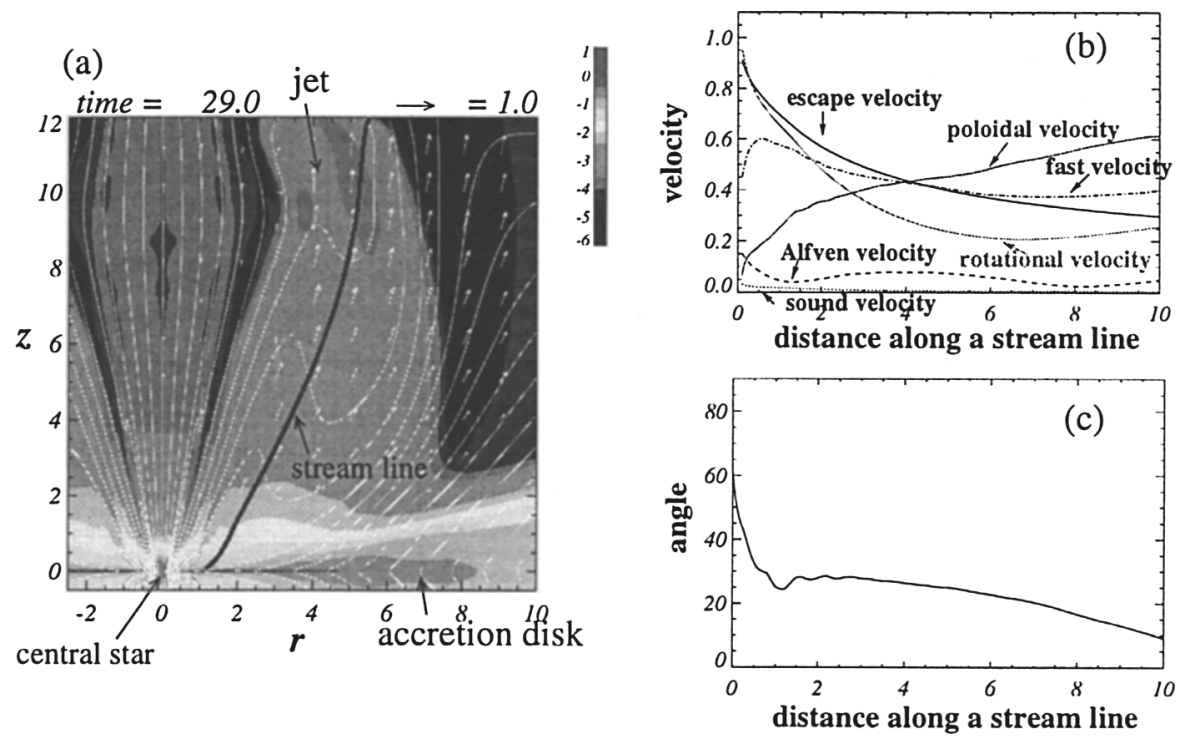

Figure 1. (a) The color density map, magnetic field lines (white lines), poloidal velocity vectors (arrows), and a stream line (a thick line). (b) The velocities along the stream line. (c) The angle between the stream line and $z$ axis.

line. The velocity along the stream line is plotted in Figure 1b. The poloidal velocity of the outflow exceeds the fast magnetosonic velocity as well as the escape velocity.

In order to show the collimation of the flow, we plotted the angle between the stream line and $z$ axis as a function of distance along the stream line (Figure 1c). The angle decreases as $z$ increases, which shows the collimation of the outflow. The outflow is collimated by the pinch effect of the toroidal component of the magnetic field that is produced by the rotation of the disk.

\section{References}

Kudoh, T., Matsumoto, R., \& Shibata, K. 1998, ApJ, 506, 186

Kudoh, T., \& Shibata, K. 1995, ApJ, 452, L41

Kudoh, T., \& Shibata, K. 1997a, ApJ, 474, 362

Kudoh, T., \& Shibata, K. 1997b, ApJ, 476, 632

Matsumoto, R., Uchida, Y., Hirose, S., Shibata, K., Hayashi, M. R., Ferrari, A., Bodo, G., \& Norman, C. 1996, ApJ, 461, 115

Shibata, K., \& Uchida, Y. 1986, PASJ, 38, 631

Uchida, Y., \& Shibata, K. 1985, PASJ, 37, 515 